\title{
On a Buffering Hypothesis in 802.11 Analytic Models
}

\author{
K. D. Huang and K. R. Duffy
}

\begin{abstract}
Using detailed statistical analysis of data taken from simulations and test-bed experiments, we have recently reported the inappropriateness of a queue-decoupling approximation that has been implicitly adopted by many distinct authors to incorporate queueing behavior in analytic models of finite load 802.11 networks. In the present paper we show that this flawed hypothesis leads to network throughput prediction errors in the presence of stations with asymmetric offered loads. We suggest that care should be taking in drawing deductions from models that adopt this hypothesis and that further modeling innovation is necessary.
\end{abstract}

Index Terms-WLAN, IEEE 802.11, performance evaluation.

\section{INTRODUCTION}

B IANCHI'S seminal papers [1][2] introduced a celebrated mean-field Markov model of saturated WLANs employing IEEE 802.11. Assuming that each station in the network always has packets to send, this modeling approach enables the accurate prediction of the long run maximum stable throughput of an 802.11 infrastructure mode network. Bianchi's methodology is based on a collision decoupling approximation. Assume that each station $i$ has a fixed probability of collision given it is attempting transmission, $p_{i}$, that does not dependent on collision history. Then the station's back-off counter can be identified as an irreducible, aperiodic Markov chain from which the stationary probability that station $i$ is attempting transmission, $\tau_{i}:=\tau_{i}\left(p_{i}\right)$, can be determined explicitly as a function of $p_{i}$, as well as station and network parameters. The self-consistent fixed point equations

$$
1-p_{i}=\prod_{j \neq i}\left(1-\tau_{j}\left(p_{j}\right)\right), \text { for each station } i,
$$

state that the probability station $i$ experiences no collision given it is attempting transmission is the probability that no other station is attempting transmission. The unique solution $\left\{p_{i}^{*}\right\}$ of the system of equations (1) determines the operating point of the network. From $\left\{p_{i}^{*}\right\}$, fundamental performance quantities, such as per-station throughput, can be predicted.

Due to its intuitive appeal and its predictive accuracy, Bianchi's approach has been widely adopted and developed for use in models that expand on its original range of applicability. For example, as stations in a typical network are not saturated, many authors have extended the paradigm to incorporate stations with finite load. For these models to be mathematically tractable, packet arrival at stations are assumed to follow a Poisson process. In these finite load models, if stations can buffer only a single packet, then no additional hypotheses beyond Bianchi's original decoupling assumption

Manuscript received January 27, 2009. The associate editor coordinating the review of this letter and approving it for publication was G. Lazarou.

The authors are with the Hamilton Institute, NUI Maynooth, Co. Kildare, Ireland (e-mail: ken.duffy@nuim.ie).

This work was supported by SFI grant RFP-07-ENEF530.

Digital Object Identifier 10.1109/LCOMM.2009.090176 are necessary (e.g. [3][4][5]). These models continue to make accurate predictions.

To analytically model stations with packet buffers, two distinct approaches have been proposed: (A) the expansion of the original model's state space to include buffer occupancy (e.g [6]); (B) the adoption of an additional queue-decoupling assumption, which we explain below. The drawback of the former approach is that the stationary distribution of the resulting Markov chain cannot be determined explicitly, so that identifying the functions $\tau_{i}\left(p_{i}\right)$ is numerically time consuming. Solving these models can take as long as the time to run a packet-level simulation. The potential difficulty with the latter methodology is that an additional approximation has been adopted and thus there is the possibility of new approximation errors. The advantage of that methodology is that $\tau_{i}\left(p_{i}\right)$ is, typically, determined as an explicit function, leading to simpler and faster numerics, giving these models a significant run-time advantage over simulations.

The queue-decoupling approximation, which is often implicitly assumed by authors, can be described as follows. Select a given station. After the $k^{\text {th }}$ successful transmission, define $Q_{k}:=0$ if the station's packet buffer is empty and $Q_{k}:=1$ if the station's packet buffer is not empty. That is, $Q_{k}=1$ if there is at least one packet awaiting transmission in the station's buffer immediately after the $k^{\text {th }}$ successful transmission and otherwise it is 0 . The probability that $Q_{k}=1$ is called the queue-busy probability. It is commonly assumed (e.g. [7][8][9][10]) that: (i) the sequence $\left\{Q_{k}\right\}$ consists of independent random variables; and (ii) the sequence $\left\{Q_{k}\right\}$ consists of identically distributed random variables that, in particular, do not depend on the back-off stage at which the most recent successful transmission was made. Rather than checking these assumptions directly, finite load models that adopt it are validated by comparing their predictions of throughput and delay with simulations, typically in symmetrically loaded networks. Using detailed statistical analysis of data from simulation [11] and experiment [12] we have recently shown, inter alia, that Bianchi's collision-decoupling hypothesis is accurate even for non-saturated stations with buffers. However, while the queue-decoupling approximation makes mathematical analysis amenable, we have found that the identically distributed hypothesis, (ii) above, is inappropriate. Figure 7 in [11] illustrates the extreme dependence of queue-busy probability on back-off stage and the failure of the queue-decoupling hypothesis. This can be readily understood: the more collisions a packet experiences, the longer its MAC service time is and, therefore, it is more likely that there is another packet awaiting service at that station upon its successful transmission.

Our aim here is to show that even though models that adopt the queue-decoupling assumption have been reported to make accurate predictions, caution is needed in using their predictions. We do this by considering a setting where a 


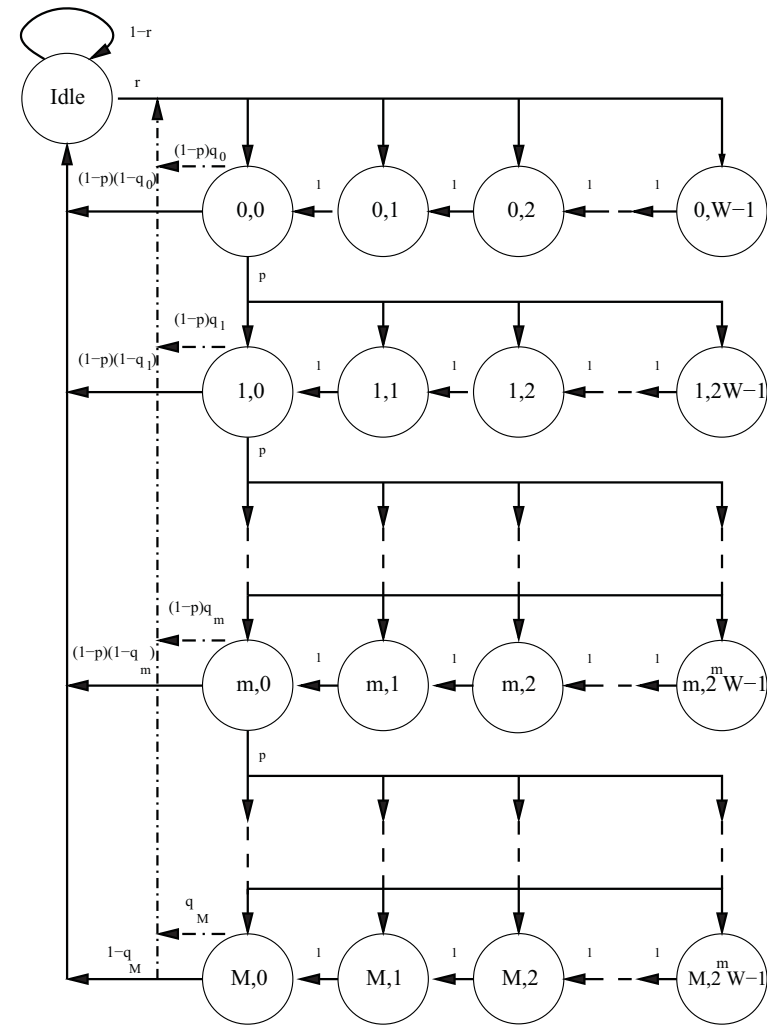

Fig. 1. Station back-off counter Markov chain.

queueing model can be built with or without the queuedecoupling approximation. In particular, we consider a model where stations can buffer one packet beyond the MAC. This enables us to show that even though the models make remarkably similar throughput predictions for symmetrically loaded networks, that under asymmetric loads, which are common in practice, they disagree.

\section{MODELS}

The basic structure of the models introduced here is similar to nearly all of those developed from Bianchi's paradigm. Model specifics where chosen as they form the simplest situation where an analytic model can be developed with or without the queue-decoupling hypothesis. Bianchi's approach is well established, so we present only key equations rather than their derivation. As in most analytic work, we assume clear channel conditions so that transmission failure is only caused by collisions and that there are no hidden stations. We also assume that there is no post back-off, so that every packet experiences at least one back-off period.

Consider a network of stations labeled $\{1, \ldots, N\}$. Let station $i$ have Poisson arrival rate $\lambda_{i}$, minimum contention window $W_{i}$, maximum contention window $2^{m_{i}} W_{i}$ and assume that packets are discarded after $M_{i}\left(\geq m_{i}\right)$ collisions. Adopting Bianchi's fundamental hypothesis, the conditional collision probability given attempted transmission $p_{i}$ is assumed not to depend on back-off stage. In addition, assume that each station can buffer a single packet in addition to the one being processed by the MAC. Temporarily suppressing the station subscript $i$, each station's back-off process is described by the Markov chain depicted in Figure 1, where the state $(k, l)$ corresponds to the current packet in the MAC having experienced $k$ collisions and having a back-off counter with value $l$. The Idle state corresponds to the station having no packet awaiting transmission. The Markov chain has the following parameters: $r$, the probability that at least one packet arrives while the station is in the idle state, $p$ the conditional collision probability and $\left\{q_{j}\right\}$ the queue-busy probability at back-off stage $j$. We will relate the $r$ and $\left\{q_{j}\right\}$ parameters to the Poisson packet arrival rate $\lambda$, depending on whether the queue decoupling assumption is adopted or not. In the general setting the stationary distribution, $b(l, k)$, of this Markov chain can be determined explicitly as a function of these parameters. In particular, defining the normalization

$$
\begin{aligned}
\frac{1}{\eta} & :=\frac{1}{2}\left(\frac{1-p^{M+1}}{1-p}+\frac{1-p-p(2 p)^{m}}{(1-p)(1-2 p)} W-\frac{2^{m} p^{M+1}}{1-p} W\right) \\
& +\frac{1-p}{r}\left(\sum_{k=0}^{M-1} p^{k}\left(1-q_{k}\right)+\frac{p^{M}}{1-p}\left(1-q_{M}\right)\right) .
\end{aligned}
$$

the stationary probability of attempted transmission is

$$
\tau\left(p, r,\left\{q_{j}\right\}\right)=\frac{1-p^{M+1}}{1-p} \eta .
$$

In order to relate the offered load parameters $r$ and $\left\{q_{j}\right\}$ we consider two models, one with and one without the queuedecoupling approximation.

Model (I): Const- $q$. Adopting the queue-decoupling approximation, $q_{j}:=q$ for all $j$ and $1-q$ is the probability that no packet arrives during an average MAC service time. With idle slots being of length $\delta(20 \mu \mathrm{s}$ in $802.11 \mathrm{~b})$ and busy slots of length $T_{b}$ (for ease of presentation, we assume that collisions and successful transmissions take the same time; $578 \mu \mathrm{s}$ plus payload at $11 \mathrm{Mbps}$ in $802.11 \mathrm{~b}$ with $\mathrm{ACK}$ header at $1 \mathrm{Mbps}$ ), the expected time between counter decrements is

$$
T:=\prod_{i=1}^{N}\left(1-\tau_{i}\right) \delta+\left(1-\prod_{i=1}^{N}\left(1-\tau_{i}\right)\right) T_{b},
$$

while the average time from when a packet starts being processed by the MAC until it successful transmission is

$$
E(B)=\frac{1}{2}\left(W \frac{1-p-(2 p)^{m}}{1-2 p}-1\right) .
$$

Based on the reasoning in [5], we use the standard approximations that $1-r=\exp (-\lambda T)$ and $1-q=\exp (-\lambda E(B) T)=$ $(1-r)^{E(B)}$, so that the final term in equation (2) reduces to $(1-r)^{E(B)} / r$. To solve the Const-q model, using (3), one searches for a solution of fixed point equations (1).

Model (II): Var-q. For the model without the queuedecoupling approximation, we wish to assume as little as possible beyond Bianchi's collision decoupling hypothesis. This leads us to treating slots being idle or busy as an i.i.d. process as well as having the queue-busy probability depend on backoff stage. Again suppressing station-based subscripts, we have that

$$
1-r=\prod_{k \neq i}\left(1-\tau_{k}\right) e^{-\lambda \delta}+\left(1-\prod_{k \neq i}\left(1-\tau_{k}\right)\right) e^{-\lambda T_{b}} .
$$




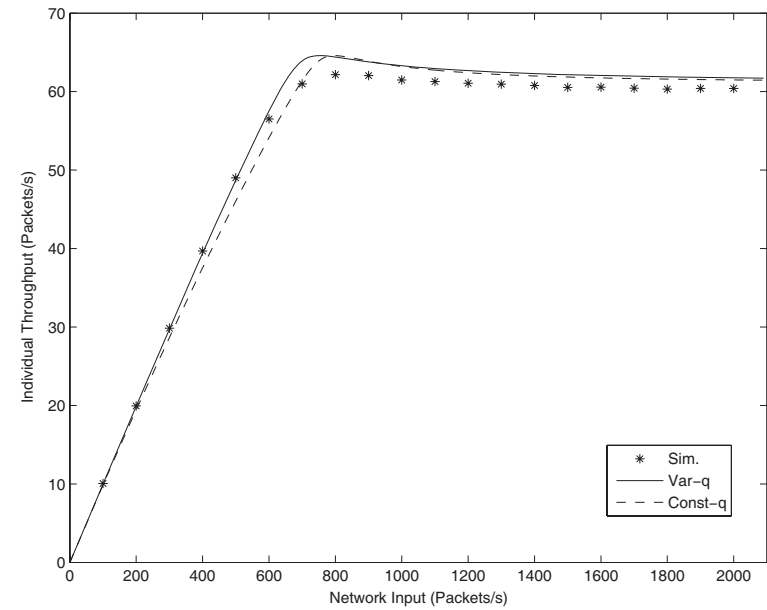

Fig. 2. Symmetric offered load. $N=10$. Predictions and ns-2 simulation.

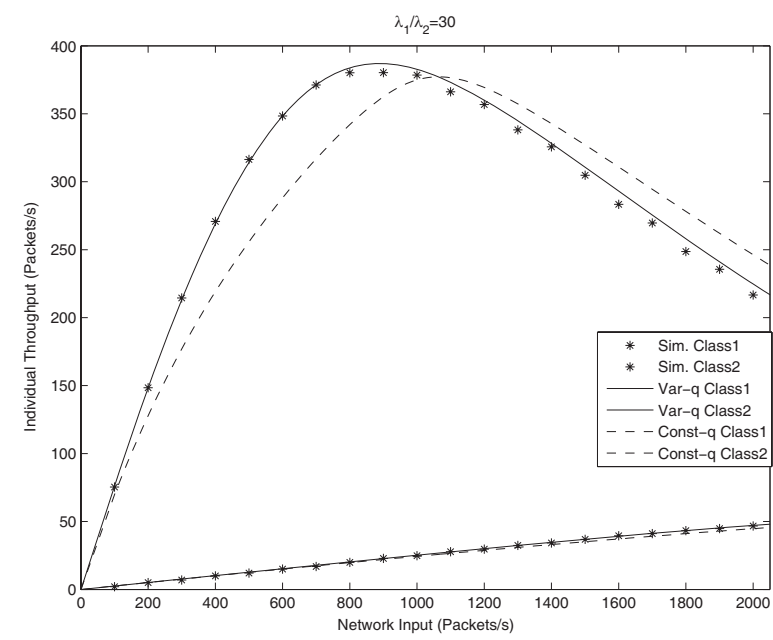

Fig. 3. Asymmetric offered load. $N=10$. One station with arrival rate $\lambda_{1}$ and nine with rate $\lambda_{2}$. Predictions and ns-2 simulation.

For $0 \leq j<m$, we have that

$$
1-q_{j}=\left(\prod_{l=0}^{j} \frac{1}{2^{l} W}\right) e^{-\lambda T_{b}(j+1)}\left(\prod_{l=0}^{j} \frac{1-(1-r)^{2^{l} W}}{r}\right)
$$

and, for $m \leq i \leq M$, we have the equality

$$
1-q_{j}=\left(1-q_{j-1}\right) \frac{1-(1-r)^{2^{m} W}}{2^{m} W r} e^{-\lambda T_{b}} .
$$

To solve the Var-q model, using (3), one searches for a solution of these equations in addition to the fixed point equation (1).

\section{RESULTS}

Consider an $802.11 \mathrm{~b}$ infrastructure mode network with $N$ stations that have $802.11 \mathrm{~b}$ parameters identical to those in Table I [5]. All packets have a 1000 byte payload. A model validation scenario of the sort commonly used by authors is where there are 10 stations and the arrival rates of all stations are the same. In Figure 2, model predictions of station throughputs are compared with the output of ns2 packet level simulations. It can be seen that the Const$\mathrm{q}$ and Var-q models are nearly inseparable and both make remarkably accurate throughput predictions when compared with results from simulations. Validation scenarios of this sort have led many authors to deduce that the queue-decoupling hypothesis is a good approximation. However, Figure 3 reports on the same network, but where the arrival rate at one station is thirty times that of the nine others: $\lambda_{1}=30 \lambda_{2}$. The predictions of the two models no longer coincide, with the Var-q model continuing to make accurate predictions, while the Const-q model does not. Other asymmetric slices through the throughput versus offered load surface for networks of various sizes, $N$, show similar differences in predictions. This indicates that the flawed queue-decoupling hypotheses does follow through to prediction inaccuracies.

\section{CONCLUSIONS}

We have shown how a commonly adopted, but flawed, hypothesis used in modeling 802.11 infrastructure model networks can lead to predictive errors. As finite load models are used, for example, to propose load-dependent $802.11 \mathrm{e}$ parameterizations to achieve fair bandwidth allocation in infrastructure and mesh networks (e.g. [13], [14]), these inaccurate predictions could lead to settings that perform poorly. This work suggests that to build an analytic model that incorporates buffering, is tractable and has run-time advantages over simulation, one must either accept the inaccuracies that come with the queue-decoupling hypothesis or the research community must rise to the challenge of providing new modeling innovation.

\section{REFERENCES}

[1] G. Bianchi, "IEEE 802.11-saturated throughput analysis," IEEE Commun. Lett., vol. 12, no. 2, pp. 318-320, 1998.

[2] G. Bianchi, "Performance analysis of IEEE 802.11 Distributed Coordination Function," IEEE J. Select. Areas Commun., vol. 18, no. 3, pp. 535-547, Mar. 2000.

[3] G.-S. Ahn, A. T. Campbell, A. Veres, and L.-H. Sun, "Supporting service differentiation for real-time and best-effort traffic in stateless wireless ad hoc networks (SWAN)," IEEE Trans. Mob. Comp., vol. 1, no. 3, pp. 192-207, 2002.

[4] M. Ergen and P. Varaiya, "Throughput analysis and admission control in IEEE 802.11a," ACM-Kluwer MONET, vol. 10, no. 5, pp. 705-716, 2005.

[5] D. Malone, K. Duffy, and D. J. Leith, "Modeling the 802.11 Distributed Coordination Function in non-saturated heterogeneous conditions," IEEE/ACM Trans. Networking, vol. 15, no. 1, pp. 159-172, 2007.

[6] K. Ghaboosi, B. Khalaj, Y. Xiao, and M. Latva-aho, "Modeling IEEE 802.11 DCF using parallel space time Markov chain," IEEE Trans. Veh. Technol., vol. 57, no. 4, pp. 2404-2413, July 2008.

[7] H. Zhai, Y. Kwon, and Y. Fang, "Performance analysis of IEEE 802.11 MAC protocols in wireless LANs," Wireless Commun. and Mobile Computing, vol. 4, no. 8, pp. 917-931, 2004.

[8] G. R. Cantieni, Q. Ni, C. Barakat, and T. Turletti, "Performance analysis under finite load and improvements for multirate 802.11," Elsivier Computer Communications, vol. 28, no. 10, pp. 1095-1109, 2005.

[9] C. G. Park, D. H. Han, and S. J. Ahn, "Performance analysis of MAC layer protocols in the IEEE 802.11 wireless LAN," Telecommun. Systems, vol. 33, no. 1-3, pp. 233-253, 2006.

[10] K. Duffy and A. J. Ganesh, "Modeling the impact of buffering on 802.11," IEEE Commun. Lett., vol. 11, no. 2, pp. 219-221, 2007.

[11] K. D. Huang, K. R. Duffy, D. Malone, and D. J. Leith, "Investigating the validity of IEEE 802.11 MAC modeling hypotheses," in Proc. IEEE PIMRC, Cannes, France, 2008.

[12] K. D. Huang, K. R. Duffy, and D. Malone, "On the validity of IEEE 802.11 MAC modeling hypotheses," technical report, NUI Maynooth, 2008.

[13] P. Clifford, K. Duffy, J. Foy, D. J. Leith, and D. Malone, "Modeling 802.11e for data traffic parameter design," in Proc. WiOPT, 2006.

[14] K. Duffy, D. J. Leith, T. Li, and D. Malone, "Modeling 802.11 mesh networks," IEEE Commun. Lett., vol. 10, no. 8, pp. 635-637, 2006. 